\title{
ANALYSIS OF DIFFERENT HIERARCHICAL ROUTING PROTOCOLS OF WIRELESS SENSOR NETWORK
}

\author{
Dipti Balkrushna Ram¹, Grishma Shaileshkumar Shah ${ }^{2}$ \\ ${ }^{1,2}$ Master of engineering, Electronics and Communication, SAL Institute of technology and engineering research, \\ Gujarat, India
}

\begin{abstract}
, Abstarct
Wireless sensored network is nowadays very popular in the field of research because world is now switching faster from wired communication to the wireless communication. It is used in environment monitoring, habitat monitoring, battlefield etc. WSN is made up of tiny sensor nodes which senses the data and communicate to the base station via other nodes.WSN networks are datacentric rather than node centric. So, main issues in WSN networks are energy consumption of network, lifetime of a network, delay, latency, quality of service etc.WSN has defined many routing protocols for the network. The main challenge in WSN is to design a routing protocol which gives the maximum energy efficient routing because nodes in sensored network are equipped with the battery. So, as time passes the battery of nodes will decrease so in turn network lifetime will decreases. There are many routing protocols which are classified as their working and their application to different conditions. This paper describes a brief information about routing protocols. The main focus of this paper is to give the comparison of different hierarchical routing protocols.
\end{abstract}

Keywords: Leach, Pegasis,Teen/Apteen, WSN

\section{INTRODUCTION}

Designing of a wireless sensored network having increased energy efficiency is the hot research area nowadays. In recent years sensored network has reach the most of fields like medical monitoring, environment monitoring, habitat monitoring, home security system etc. The future is not for any when the wireless sensor network will be spreaded over the large area of the world. Because it is an emerging technology, at present there are lots of things in WSN which needs the attention by the researchers. There are many models which have been introduced and applied to wireless sensored network for better energy efficiency, network lifetime, delay, latency, etc. WSN have data centric architecture compared to ad-hoc network which consist of node centric architecture. In WSN there is no IP address scheme for each node because it has densely deployed sensor nodes in its large area. Due to these unique features of WSN protocol design is very important parameter in WSN. By designing proper routing protocol one can utilize the limited resources of the network.

There are many types of routing protocols in wireless sensor network, which are classified as follows:

1. Flooding and gossiping

Flooding and gossiping is the basic routing protocols of WSN. In flooding each node broadcast the receiving packets to all its neighbour and this process is continued until the packets has been reached to its destination.

2. Directed diffusionIt uses the attribute value pairs of the data and queries the sensors in an on demand basis. It diffuses data through sensor nodes by using a scheme of naming to the data.

\section{Rumour routing}

It is similar to the directed diffusion but it is mostly applicable where geographic routing conditions are not preferred.

\section{Gradient based routing}

It is also a changed version of directed diffusion.

In GBR the number of hopes when node is diffused in the network is recorded. So, each node will discover the minimum number of hops to the sink which is known as the height of that node.

"The difference between node's height and that of its neighbour is called gradient on that link." ${ }^{, 2]}$

And the packet is forwarded on a link which has largest gradient.

\section{Energy aware routing}

Energy aware routing is based on the stochastic model.

In this scheme the set of sub optimal path is used to increase the lifetime of a network.

It says that using minimum energy path every time will dissipate the energy of nodes on that path. Instead, we can use multiple paths with some probability which in turn increase the lifetime of the network.

6. Hierarchical protocols

Network scalability is the major issue in designing a wireless sensor network. 
As the network area and nodes density increases there may be overload in single tire network. This overload causes latency and unnecessary tracking of events. Other thing is that single tire network is not scalable for a large set of sensors in large area and there is a problem of service degradation. To overcome all these problems the hierarchical routing approach is used in some of the protocols of WSN. It maintains the energy consumption of the nodes using multi hop communication forming a cluster of nodes. LEACH (lower energy adaptive clustering hierarchy) is the first routing protocol invented for the hierarchical routing.

This routing protocol has inspired many other hierarchical routing protocols like PEGASIS, TEEN, APTEEN, etc. In the next section we will explore the different hierarchical routing protocols.

\section{HIERARCHICAL ROUTING PROTOCOLS}

\subsection{Leach}

LEACH is the first hierarchical routing protocol used for wireless sensored network. LEACH uses adaptive clustering scheme for the communication between nodes. It uses TDMA scheme for the timeline operation LEACH can prolong the network lifetime with a proportion of $15 \%$. LEACH forms the clusters head. The nodes which want to communicate with other nodes will not directly communicate but it will be communicated via cluster heads. In each TDMA frame in each round the cluster head nodes will be changed. The selection of cluster heads will be done by the following equation:

$$
T(n)=\frac{\mathrm{p}}{1-\mathrm{p} \cdot\left(\operatorname{rmod} \frac{1}{\mathrm{p}}\right)}, \mathrm{n} \in \mathrm{G}
$$

Where $\mathrm{P}=$ percentage of cluster heads

$\mathrm{R}=$ the current round

$\mathrm{G}=$ set of nodes that haven't been cluster heads in the last $1 / \mathrm{P}$ rounds.

Here, $T(n)$ is the threshold value of the selection of cluster heads in each round. Cluster heads are produced according to this equation the sensor node generate a random number between 0 and 1 , if the number is smaller than the threshold
$\mathrm{T}(\mathrm{n})$, then the node is selected as cluster head

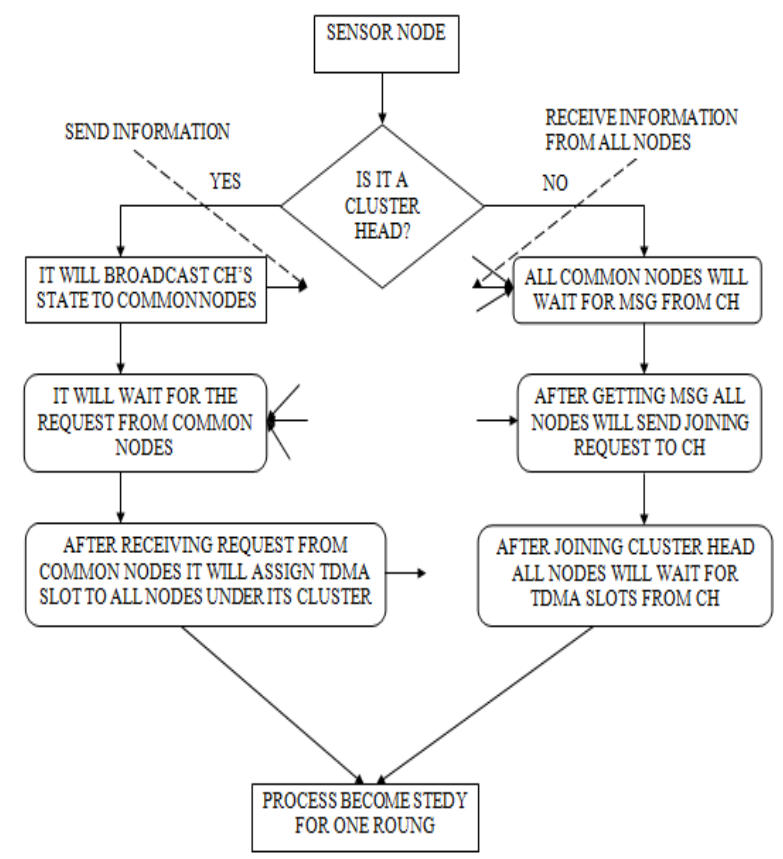

Fig-1: Flow chart for $\mathrm{CH}$ selection

As shown in figure the node will become cluster head when its energy is higher than the energy of other nodes. The $\mathrm{CH}$ node will send the joining request message to all common nodes in its cluster. The common nodes will decide to join or not the $\mathrm{CH}$. After getting acknowledgement from common nodes $\mathrm{CH}$ will assign TDMA scheme to all nodes.

In this way the LEACH protocol will work using clustering scheme. Some features of LEACH protocol is as follows.

1. LEACH protocol randomly selects cluster heads in each round. So, to become $\mathrm{CH}$ using quickly some nodes will dissipate energy very fast to be selected as a cluster head more times.

2. In LEACH cluster heads directly communicate with sink. So, the energy consumption between $\mathrm{CH}$ and sink in greater than the energy consumption of communication between CH's.

3. LEACH has great improvement in energy consumption great improvement in energy consumption of direct communication because it uses clustering hierarchy and also it is not a single tire communication as direct diffusion scheme or gossiping and flooding scheme.

4. Though LEACH has improved the energy consumption, further improvement is needed as the unbalanced energy load and large unnecessary energy dissipation. It is caused by the randomicity in electing cluster-head nodes.

5. There is an extra energy dissipation, because in each round nodes acting as $\mathrm{CH}$ will consume a lot of energy to transmit data to far distance base station. 


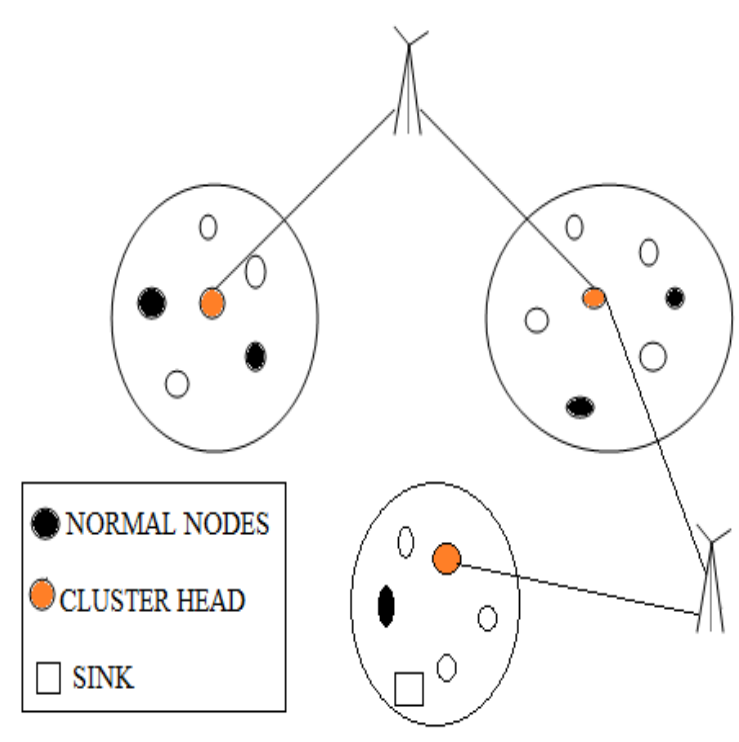

Fig-2: architecture of LEACH protocol

\subsection{Pegasis}

The improvement of LEACH protocol is PEGASIS (power efficient gathering in sensor information system). It makes the chain of nodes instead of making clusters of it. The farther node will transmit the data via its neighbour node and in this way chain is created. The last node in the chain is called the leader node which will transmit all the data to sink (base station).

\section{LEADER NODE}

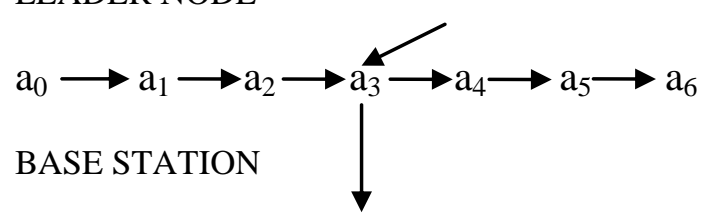

Fig-3: PEGASIS protocol

As shown in figure the nodes $\mathrm{a}_{0}$ to $\mathrm{a}_{6}$ are the chain partners for data transmission. Here $a_{3}$ is the leader node which will transfer all the data to base station (sink).

Features of PEGASIS

1. PEGASIS uses multihop routing by forming chains and use only one node to transmit to the Base station.

2. Compare to LEACH protocol PRGASIS has been shown performance of about $100-300 \%$ for different network topologies of WSN.

3. Compare to LEACH protocol the lifetime of a PEGASIS is long.

4. In PEGASIS there is only one node which does the data aggregation and data fusion. So, compared to LEACH where each cluster head is taking part in communication with base station. So, in turn the energy will also be dissipated by each cluster head, PEGASIS will dissipate less energy because only leader node in chain will actively take part in data aggregation and data fusion.

5. In PEGASIS each node should be aware of the remaining energy status of its neighbours.
6. Compare to LEACH, PEGASIS removes the energy dissipated by the reformation of cluster in each round.

7. In PEGASIS, if node death occurs, the chain must be rebuilt which increases the energy consumption.

8. Node far away from the leader node will forward the data many times by the chain which causes the long time delay. So, there is a problem of time delay in PEGASIS protocol which should be improved.

\subsection{TEEN and APTEEN (Threshold sensitive}

\section{energy efficient sensor network)}

These protocols are bit different from LEACH and PEGASIS protocols because it uses the sensored attributes such as temporary for communication. These protocols are very useful in time critical applications, where wireless network is reactive. In LEACH and PEGASIS the network is proactive where there is no event driven things occur where as in reactive network the things which are event driven which are sensitive to nodes sensitivity like temperature, weather or any other environmental conditions. TEEN uses hierarchical network architecture with data centric mechanism. The figure shows the mechanism of cluster forming in TEEN and APTEEN. As shown in figure clusters are formed and this process of formation of cluster is continued to the second level till the base station (sink) is reached. There are two types of thresholds: hard and soft. After formation of cluster head's the cluster head will broadcast the threshold to the common nodes in its clusters. The first threshold sent to all nodes by cluster head is hard threshold which is the minimum value of sensory attributes to trigger node for switching on its transmitter. So, transmission occurs only when the node's sensed attribute in turn lower the number of transmissions. Once, the node had sensed a value beyond hard threshold, it only transmits a data when value of sensed attribute changes by an amount equal or greater than the soft threshold. So, soft threshold will also decreases the number of transmissions.

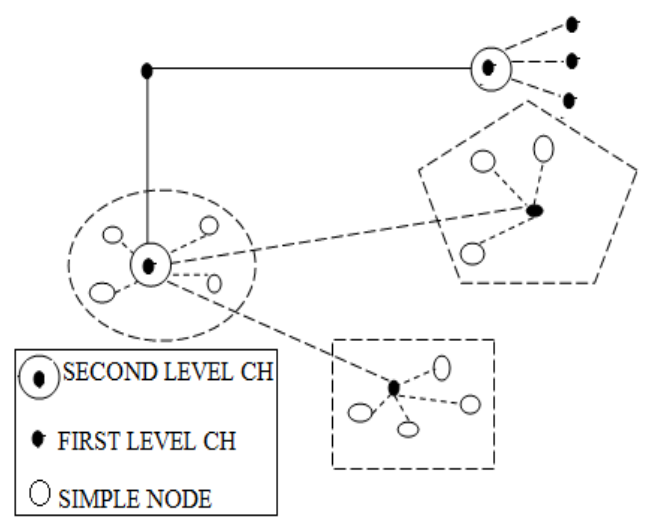

Fig-4:TEEN/APTEEN protocol architecture

APTEENC Adaptive threshold sensitive energy efficient sensor network protocol is extension of TEEN.

Features of TEEN and APTEEN

1. TEEN is not applicable where periodic reports are needed because it is based on thresholds. 
2. The APTEEN gives the facilities which TEEN does not gives as follows:

$$
\begin{aligned}
& >\text { Analysing past data values } \\
& >\quad \text { Taking snapshot view of the network. } \\
& >\quad \text { To monitor an event for a period of time. }
\end{aligned}
$$

3. In APTEEN cluster head's broadcast the attributes the threshold values and schedule of transmission of all nodes.

4. TEEN and APTEEN gives better performance than LEACH. Energy dissipation of APTEEN is in between of LEACH and TEEN.

5. Compared to LEACH, TEEN/APTEEN has overhead and complexity of cluster formation and problem of giving threshold based functions.

\section{CONCLUSION AND FUTURE SCOPE}

From the analysis of the different routing protocols we have shown that there is further need of improvement in LEACH protocol as compared to PEGASIS and TEEN/APTEEN protocols. The table shown below gives the comparison of

\begin{tabular}{|c|c|c|c|}
\hline PARAMETER & LEACH & $\begin{array}{c}\text { PEGASI } \\
\mathrm{S}\end{array}$ & $\begin{array}{c}\text { TEEN/APTEE } \\
\mathrm{N}\end{array}$ \\
\hline $\begin{array}{c}\text { DATA } \\
\text { DELIVERY } \\
\text { MODEL } \\
\end{array}$ & $\begin{array}{l}\text { CLUSTE } \\
\text { R HEAD }\end{array}$ & $\begin{array}{l}\text { CHAIN } \\
\text { BASED }\end{array}$ & $\begin{array}{c}\text { ACTIVE } \\
\text { THRESHOLD }\end{array}$ \\
\hline $\begin{array}{c}\text { DATA } \\
\text { AGGREGATIO } \\
\mathrm{N}\end{array}$ & YES & $\mathrm{NO}$ & YES \\
\hline $\begin{array}{l}\text { POWER } \\
\text { USAGE }\end{array}$ & $\mathrm{HIGH}$ & MAX & $\mathrm{HIGH}$ \\
\hline SCALABILITY & GOOD & GOOD & GOOD \\
\hline $\begin{array}{l}\text { QUALITY OF } \\
\text { SERVICE }\end{array}$ & $\mathrm{NO}$ & $\mathrm{NO}$ & GOOD \\
\hline $\begin{array}{l}\text { NETWORK } \\
\text { LIFETIME }\end{array}$ & GOOD & $\begin{array}{l}\text { VERY } \\
\text { GOOD }\end{array}$ & VERY GOOD \\
\hline $\begin{array}{c}\text { RESOURCE } \\
\text { AWARENESS }\end{array}$ & YES & YES & YES \\
\hline
\end{tabular}
these four protocols.

Table-1: Protols and their Parameters

From chart-1 shown below we can see that when first node dies the energy dissipation of LEACH is very much poor, so in terms of energy dissipation and network lifetime there is a lot more to do research work. Also researchers can futher make improvements in PEGASIA in terms of time delay. There are many open issues in WSN for improving the routing algorithms of LEACH, PEGASIS, TEEN/APTEEN.

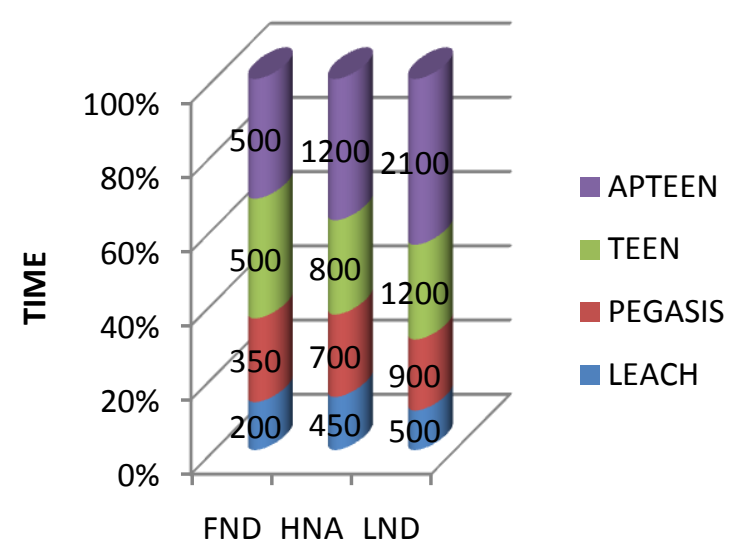

Chart-1:Dead nodes VS Lifetime of nodes

\section{REFERENCES}

[1] Kemal Akkaya, Mohamed Younis, “A survey on routing protocols for wireless sensor networks", 26 November 2003. [2] Tintu Devasia, Gopika S, "Statistical Analysis of Energy Efficient Hierarchical Routing Protocols in WSN", International Journal of Advanced Research in Computer and Communication Engineering, Vol. 2, Issue 5, May 2013.

[3] Fan Xiangning, Song Yulin, "Improvement on LEACH Protocol of Wireless Sensor Network", 2007 International Conference on Sensor Technologies and Applications, IEEE.

[4] $\mathrm{Mu}$ Tong, Minghao Tang, "LEACH-B:An Improved LEACH Protocol for Wireless Sensor Network", 978-14244-3709, 2010 IEEE.

[5]Jindal V, Verma AK And Bawa S, "How The Two Adhoc Networks Can Be Different: Manet \& WSNs", International Journal Of Computer Science \& Technology, 2011, 2(4), 122-126.

[6]Wang J, yang G and Chen S,"Secure Leach Routing Protocol Based On Low-Power Cluster-Head Selection Algorithm For Wireless Sensor Networks", International Symposium On Intelligent Signal Processing And Communication Systems, 2007, 341-344.

[7]Liu Y, Gao J, Jia Y and Zhu L, "A Cluster Maintenance Algorithm Based on LEACH-DCHS Protoclol", International Conference on Networking, Architecture, and Storage, 2008, 165-166.

[8]Junping h, Yuhui J and Liang D, “A Time-based ClusterHead Selection Algorithm for LEACH", In Computer and Communication, 2008.

[9]Zhixiang D and Bensheng Q, "Three-layered routing protocol for WSN based on LEACH algorithm", IEEE Xplore, 2007, 72-75.

[10]Tong $\mathrm{M}$ and tang $\mathrm{M}$, "LEACH-B: An Improved LEACH Protocol for Wireless Sensor Network", $6^{\text {th }}$ International Conference on Wireless Communications Networking and Mobile Computing, 2010.

[11]Heinzelman WB, Chandraskan PA and Blakrisshnan $\mathrm{H}$, "An Application-Specific Protocol Architecture for Wireless 
Microsensor Networks", IEEE Trans. on Wireless

Communication, 2002, 1(4), 660-670.

[12]Zhi-Jun L and La-Yuan L, "Based on Energy Balance LEACH-DC Protocol Design", $6^{\text {th }}$ IEEE Joint International Conference on Information Technology and Artificial Intelligence Conference (ITAIC), 2011.

[13]Kumar N And Kaur J, "Improved LEACH Protocol for Wireless Sensor Networks", $7^{\text {th }}$ International Conference on Wireless Communications, Networking and Mobile omputing (WiCOM), 2011.

[14]Tang L and Liu S, "Improvement on LEACH Routing Algorithm for Wireless Sensor Networks", International Conference on Internet Computing and Information Services, 2011.

[15] Sunita Rani, Tarun Gulati, “ An improved PEGASIS protocol to enhance energy utilization in wireless sensor network", International Journal of Advanced Research in computer science and software engineering, volume:2, Issue 11. November,2012. 